\title{
Analysis of Variation in Anthocyanin \\ Composition in Korean Coloured Potato \\ Cultivars by LC-DAD-ESI-MS and PLS-DA
}

\author{
Heon Woong Kim ${ }^{1}$ • Sung Ryong Kim² ${ }^{2}$ \\ Young Min Lee ${ }^{3} \cdot$ Hwan Hee Jang ${ }^{1}$. \\ Jung Bong Kim ${ }^{1}$
}

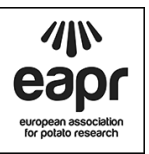

Received: 23 July 2015 / Accepted: 12 September 2017 /

Published online: 21 November 2017

C The Author(s) 2017. This article is an open access publication

\begin{abstract}
Anthocyanin concentration and composition and the effect of steaming and baking on these were evaluated in tubers of Korean red- and purple-fleshed potato cultivars and breeding clones using liquid chromatography with diode array detection and electrospray ionization-mass spectrometry (LC-DAD-ESI-MS). Twenty-six anthocyanins were isolated, of which 24 were identified. Remarkably, five cis isomers were identified, of which four, viz., cis-petanin, cis-peonanin, petunidin 3-ciscaffeoylrutinoside-5-glucoside, and petunidin 3-cis-feruloylrutinoside-5-glucoside, are reported for the first time. Moreover, pelargonidin 3-p-coumaroylrutinoside-5-glucoside (pelanin), peonidin 3-p-coumaroylrutinoside-5-glucoside (peonanin) and petunidin 3- $p$-coumaroylrutinoside-5-glucoside (petanin) were identified as the principal anthocyanins. We found that the total anthocyanin content of coloured potatoes was decreased by steaming and baking compared with the raw state. In addition, we performed partial least square discriminant analysis (PLS-DA) to discriminate between the analyzed anthocyanins. $C$ is isomers seemed to play a vital role as a biomarker in the PLS-DA model based on the type of processing and colour of the tubers.
\end{abstract}

Keywords Chemical classification · Cis-trans isomer $\cdot$ Food-processed forms $\cdot$ Mass spectrometry

Jung Bong Kim

jungbkim@korea.kr

1 National Institute of Agricultural Sciences, Rural Development Administration, Wanju, Jeollabuk-do 54875, Republic of Korea

2 Agricultural Research \& Extension Services, Jeju Special Self-Governing Province, Jeju 690-909, Republic of Korea

3 Department of Food and Nutrition, Seoul Women's University, Seoul 139-774, Republic of Korea 


\section{Introduction}

Anthocyanins, a class of flavonoids, are natural water-soluble pigments mainly found in vegetables and fruits. In nature, thousands of anthocyanins have been identified, whose structures differ in the type and number of sugar units, organic acids, and phenolic acids (Castaneda-Ovando et al. 2009). Anthocyanins possess attractive pharmacological profiles, with anti-cancer, anti-oxidant, anti-inflammatory, apoptosis-inducing, and cell proliferation-inhibiting properties (Kong et al. 2003; Wang and Stoner, 2008). Kalt et al. (2008) showed that intact anthocyanins of the monoglycoside type can accumulate in tissues including the liver, eye, cortex, and cerebellum of blueberryfed pigs, suggesting a capacity to absorb anthocyanins.

Potatoes are the fourth most important food crop in the world. They are rich in starch and are being consumed as a staple food in many countries. Although potatoes contain a wide variety of compounds such as vitamin $\mathrm{C}$, carotenoids, chlorogenic acid, and carbohydrates, they have not been recognized as an anti-oxidant food because of their high calorie content (Ezekiel et al. 2011). However, coloured potatoes play a vital biological role in humans because of their highly bioactive anthocyanins (Zhao et al. 2009). Anthocyanins from Korean coloured potatoes have received much attention due to their significant biological activities such as anti-oxidant, anti-mutagenic, and anticancer activities (Park et al. 2007; Park et al. 2008; Kang and Choung 2008).

When colored potatoes are boiled in water or heated in a microwave, there is a slight change in their phenolic acid and anthocyanin contents according to Mulinacci et al. (2008). The total phenolic acid content of red potatoes is reported to decrease more when boiled than when baked (Xu et al. 2009), and the anthocyanin content of purple potatoes decreases at high temperatures $\left(100-150{ }^{\circ} \mathrm{C}\right)$ (Nayak et al. 2011). In contrast, some red- and purple-fleshed potatoes show higher anthocyanin contents when boiled, steamed, baked, or microwaved than when raw (Lachman et al. 2012). Under low temperature $\left(2^{\circ} \mathrm{C}\right)$ and dark conditions, coloured potatoes have a higher pigment stability than at room temperature (Rodriguez-Saona et al. 1999; Park et al. 2004).

The Korean coloured potato cultivars 'Hongyoung' and 'Jayoung' have been bred by crossing the cultivar 'AG 34314' with the cultivar 'Daeseo' which is mostly used for potato crisp processing, and hence, cultivars Hongyoung and Jayoung were recommended nationwide for their functional edible pigments in 2007. However, very few qualitative or quantitative studies have been carried out on the anthocyanin content of these potato cultivars. It is essential to determine the individual anthocyanins available as selection criteria in breeding. There is therefore an urgent need to create a chemical library of potato anthocyanins that holds all known information regarding individual anthocyanins and their identification.

Generally, the analysis of anthocyanin content and composition involves mass spectrometry methods (Giusti et al. 1999). There are several reports on anthocyanin evaluation in various coloured potato germplasms (breeding lines and cultivars) using LC-MS methods (Brown et al. 2003; Lachman et al. 2009; Hillebrand et al. 2009; Ieri et al., 2011). These techniques enable a high degree of peak separation and reproducibility with only a small amount of sample, as well as the implementation of rapid largescale screening for the evaluation of coloured potatoes. Recently, 22 anthocyanins and 167 genes involved in the regulation of anthocyanin biosynthesis have been identified in tubers of the Korean potato cultivars Hongyoung and Jayoung using UPLC-Q-TOF- 
MS (Cho et al. 2016). Previously, quantification was limited to specific anthocyanins according to the availability of standard samples. However, using this advanced method, it is possible to quantify all individual anthocyanins by the use of an internal standard.

Hence, in the present investigation, a chemical library of potato anthocyanins was compiled from previously published analytical data and was used for identification of acylated anthocyanins using mass spectrometric data from tubers of Korean red- and purple-fleshed potatoes. Anthocyanin changes resulting from steaming and baking processes were evaluated and classified according to chemical patterns using multivariate analysis.

\section{Materials and Methods}

\section{Materials}

For this study, the Korean red-fleshed potato cultivar Hongyoung, the purple-fleshed potato cultivar Jayoung, and the purple-fleshed potato breeding clones JJe08-11, DJ12X-5 and JJe08-43 (obtained in 2010 from Jeju Special Self-Governing Province Agricultural Research \& Extension Services) were subjected to three types of processing, i.e., raw, steamed $\left(10 \mathrm{~min}\right.$ at $\left.121^{\circ} \mathrm{C}\right)$, and baked $\left(40-50 \mathrm{~min}\right.$ at $\left.200{ }^{\circ} \mathrm{C}\right)$. The samples were freeze-dried and finely ground in a sample mill for use as analytical samples and stored in a deep freezer at $-70^{\circ} \mathrm{C}$.

\section{Instrumentation and Reagents}

The instruments used for the extraction process included a refrigerated multipurpose centrifuge (Hanil Science Industrial Co. Ltd., Korea) and a shaker (JEIO TECH Co. Ltd., Korea). Malvidin 3-glucoside (Extrasynthese, France) was used as an internal standard. The HPLC reagents were water, methanol, ethyl acetate, and acetonitrile from Sigma (St. Louis, MO).

\section{Anthocyanin Extraction and Purification}

Anthocyanin extraction was carried out at low temperature for a short period to minimize loss, using a modified version of the method of Fossen et al. (2003). For quantitative analysis, $1 \mathrm{~g}$ of powdered sample was extracted with $10 \mathrm{ml}$ of $0.5 \%$ trifluoroacetic acid (TFA) in $\mathrm{MeOH}$ for $1 \mathrm{~h}$ at $4{ }^{\circ} \mathrm{C}$ in a shaker and then centrifuged $\left(3000 \mathrm{rpm}, 20 \mathrm{~min}, 4{ }^{\circ} \mathrm{C}\right)$. The supernatant solution was collected from the centrifuged sample, the residue was re-extracted twice more under the same conditions, and $5 \mathrm{ml}$ of the solution was collected from the combined extract and concentrated using $\mathrm{N}_{2}$ gas and then re-dissolved in $1 \mathrm{ml}$ of $5 \%$ formic acid in $\mathrm{H}_{2} \mathrm{O}$. A Sep-Pak $\mathrm{C}_{18}$ cartridge was flushed with $2 \mathrm{ml}$ of $\mathrm{MeOH}$, followed by the addition of $2 \mathrm{ml}$ of $\mathrm{H}_{2} \mathrm{O}$ for activation. After loading, $1 \mathrm{ml}$ of concentrated crude extract was washed with $2 \mathrm{ml}$ of $\mathrm{H}_{2} \mathrm{O}$ and eluted with $1 \mathrm{ml}$ of $\mathrm{MeOH}$. The anthocyanin filtrate was eluted and concentrated using $\mathrm{N}_{2}$ gas then re-dissolved in $1 \mathrm{ml}$ of $5 \%$ formic acid in $\mathrm{H}_{2} \mathrm{O}$ prior to analysis with HPLC-DAD. For 
identification of anthocyanins, $10 \mathrm{~g}$ of powdered sample was used and the concentrated crude extract was purified by partitioning against ethyl acetate (five times). Other conditions were the same as for the quantitative analysis, and the prepared samples were analyzed by liquid chromatography with diode array detection and electrospray ionization/mass spectrometry method (LC-DAD-ESI-MS).

\section{Quantitative and Qualitative Analyses of Anthocyanins by HPLC-DAD and LC-DAD-ESI-MS}

Anthocyanins in red- and purple-fleshed potatoes were identified and quantified using a Micromass ZQ MS (Waters Co., Milford, MA) and an Alliance e2695 HPLC system (Waters Co.) equipped with a 2998 Photodiode Array (PDA) detector. In addition, a Synergi Polar-RP 80A reversed-phase column $(4.6 \times 250 \mathrm{~mm}, 4 \mu \mathrm{m}$; Phenomenex, Torrance, CA) was used. The analysis was conducted at a flow rate of $1 \mathrm{ml} / \mathrm{min}$ in the detection wavelength of $250-600 \mathrm{~nm}$ (a representative wavelength of $525 \mathrm{~nm}$ ) in a $30{ }^{\circ} \mathrm{C}$ oven. The mobile phases used were $5 \%$ formic acid in water (phase A) and $5 \%$ formic acid in water/acetonitrile $(1: 1, v / v)$ (phase B) (Kim et al. 2012). The pretreated sample was analysed using the following gradient conditions: a gradient of 20-50\% B over a 30 -min period, $40 \%$ B for $5 \mathrm{~min}$, a gradient of 40 to $20 \%$ B for $5 \mathrm{~min}$, and then a final wash with $20 \%$ B for 15 min. MS analysis was run in positive ionization mode using an electrospray ionization (ESI) source, and the MS parameters were as follows: cone voltage $30 \mathrm{~V}$, source temperature $120{ }^{\circ} \mathrm{C}$, desolvation temperature $500{ }^{\circ} \mathrm{C}$, and desolvation $\mathrm{N}_{2}$ gas flow $1020 \mathrm{l} / \mathrm{h}$. The range of molecular weights was $m / z, 200-1200$ in full-scan mode.

\section{LC-MS Library for Qualitative Analysis of Anthocyanins}

Based on a variety of literature sources, an LC-MS library of 24 anthocyanins in redand purple-fleshed potatoes was created and used for efficient determination of individual components.

\section{Multivariate Statistical Analysis (Partial Least Square Discriminant Analysis)}

By arranging and normalizing all quantitative information, a data matrix was made and $\log _{10}$-transformed data were used for multivariate statistical analysis. Partial least square discriminant analysis (PLS-DA) models were generated using SIMCA-P 11.0 (Umetrics, Umea, Sweden).

\section{Results and Discussion}

\section{Anthocyanin Diversity in the Tubers of Korean Red- and Purple- Fleshed Potatoes}

A chemical library of 24 potato anthocyanins was compiled from published potato germplasm data, containing their molecular formulas and weights, chemical names and structures, MS fragment ion patterns, UV spectra, plant resources, and references. This was used for identification of anthocyanins in the Korean-produced colored potatoes. 
The structures of individual anthocyanins from red- and purple-fleshed potatoes were identified based on the mass fragmentation patterns of rhamnoside $(\mathrm{m} / \mathrm{z}, 146)$, glucoside $(\mathrm{m} / \mathrm{z}, 162)$, rutinoside $(\mathrm{m} / \mathrm{z}, 308), p$-coumaric acid $(\mathrm{m} / \mathrm{z} 164)$, caffeic acid $(\mathrm{m} / \mathrm{z}, 180)$, and ferulic acid ( $\mathrm{m} / \mathrm{z}$ 194), acylated as hydroxycinnamic acids (Mulinacci et al. 2008; Hillebrand et al. 2009; Ieri et al. 2011). These anthocyanins were found to possess a basic structure of 3-rutinoside or 3-rutinoside-5-glucoside, which is composed of primary aglycones (i.e., pelargonidin, cyanidin, peonidin, delphinidin, petunidin, and malvidin) in mono-acylated form with $p$-coumaric acid, caffeic acid, and ferulic acid (Fig. 1).

Table 1 shows the MS fragmentation patterns of the individual anthocyanins isolated from raw and processed forms (steamed and baked) of Korean red- and purple-colored potatoes. Twenty-six anthocyanins were identified, of which 18 were consistent with the library created in this study and 8 were new, including 2 non-acylated anthocyanins, 4 cis isomers of acylated anthocyanins, and 2 presumed isomers. These isolated and identified anthocyanins displayed regular chemical patterns according to their acylation groups, and the isomers which are rarely found in raw potatoes provided relatively accurate, clear MS and UV patterns due to their increased content when baked or steamed. As a result, upon the conversion of trans isomers into cis isomers, a rotation of $140^{\circ}$ was found in the $\beta$-position of the hydroxycinnamic acid structure (Azuma

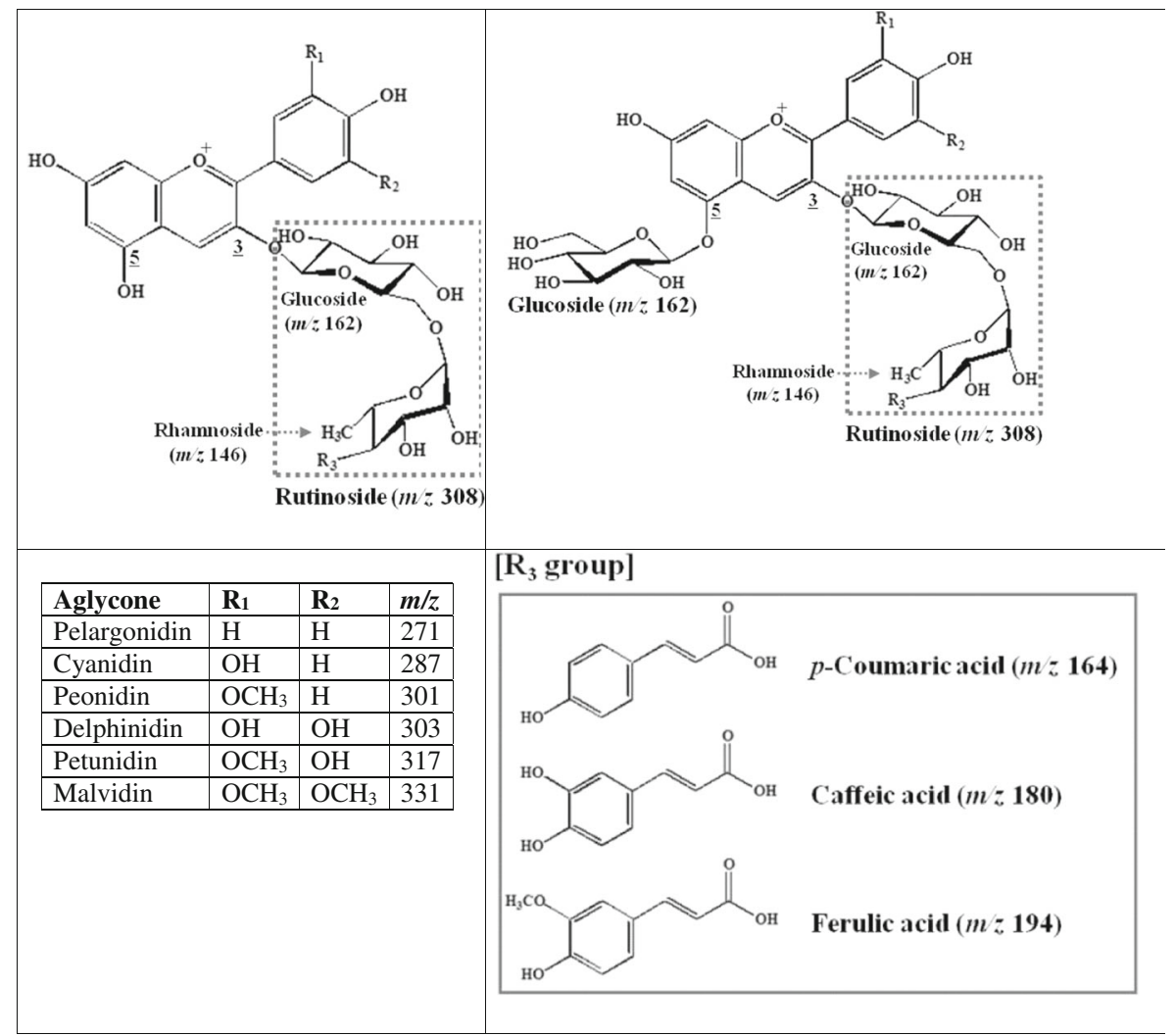

Fig. 1 Chemical structures of anthocyanins isolated from Korean red- and purple-fleshed potato extracts 


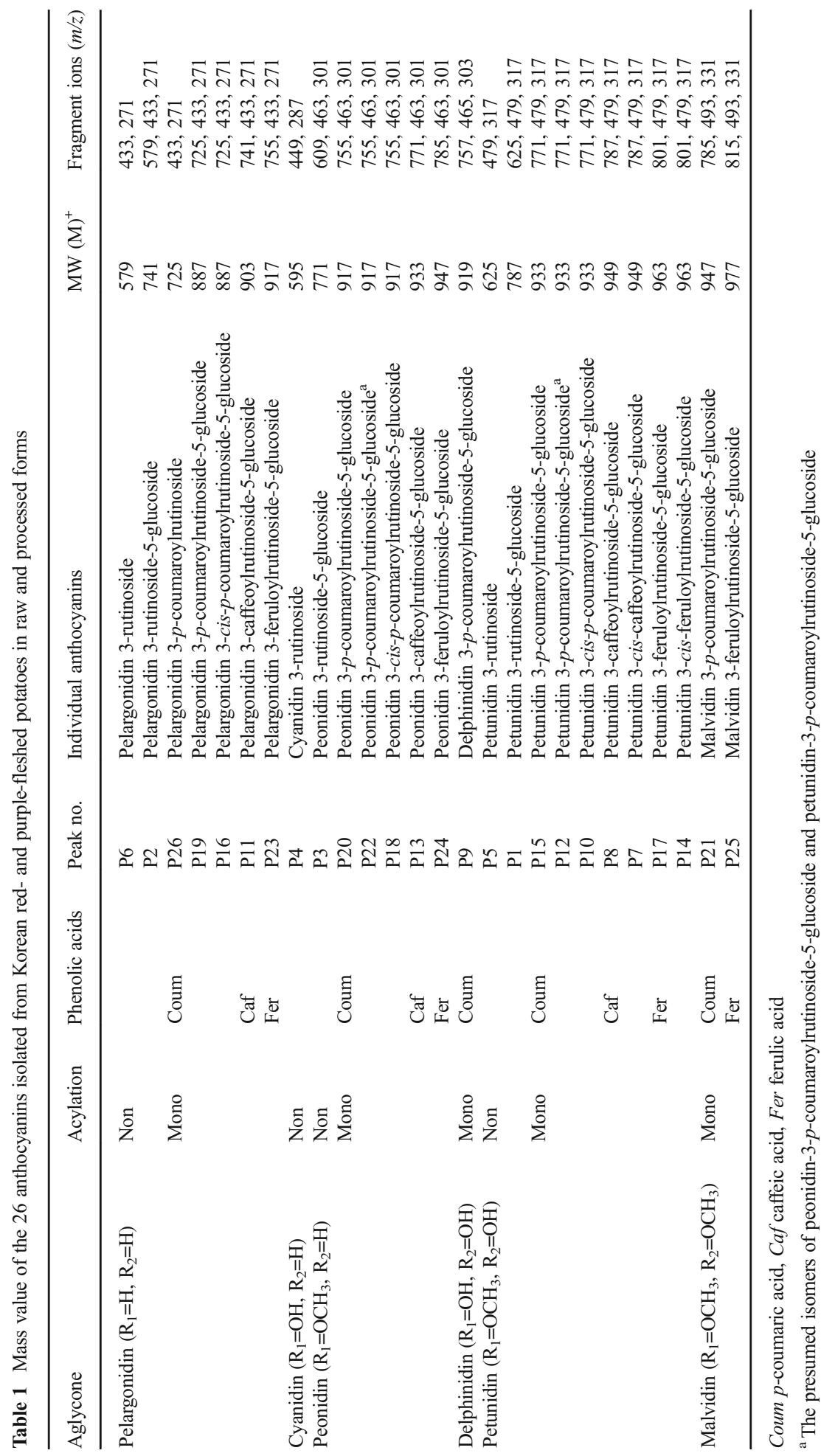




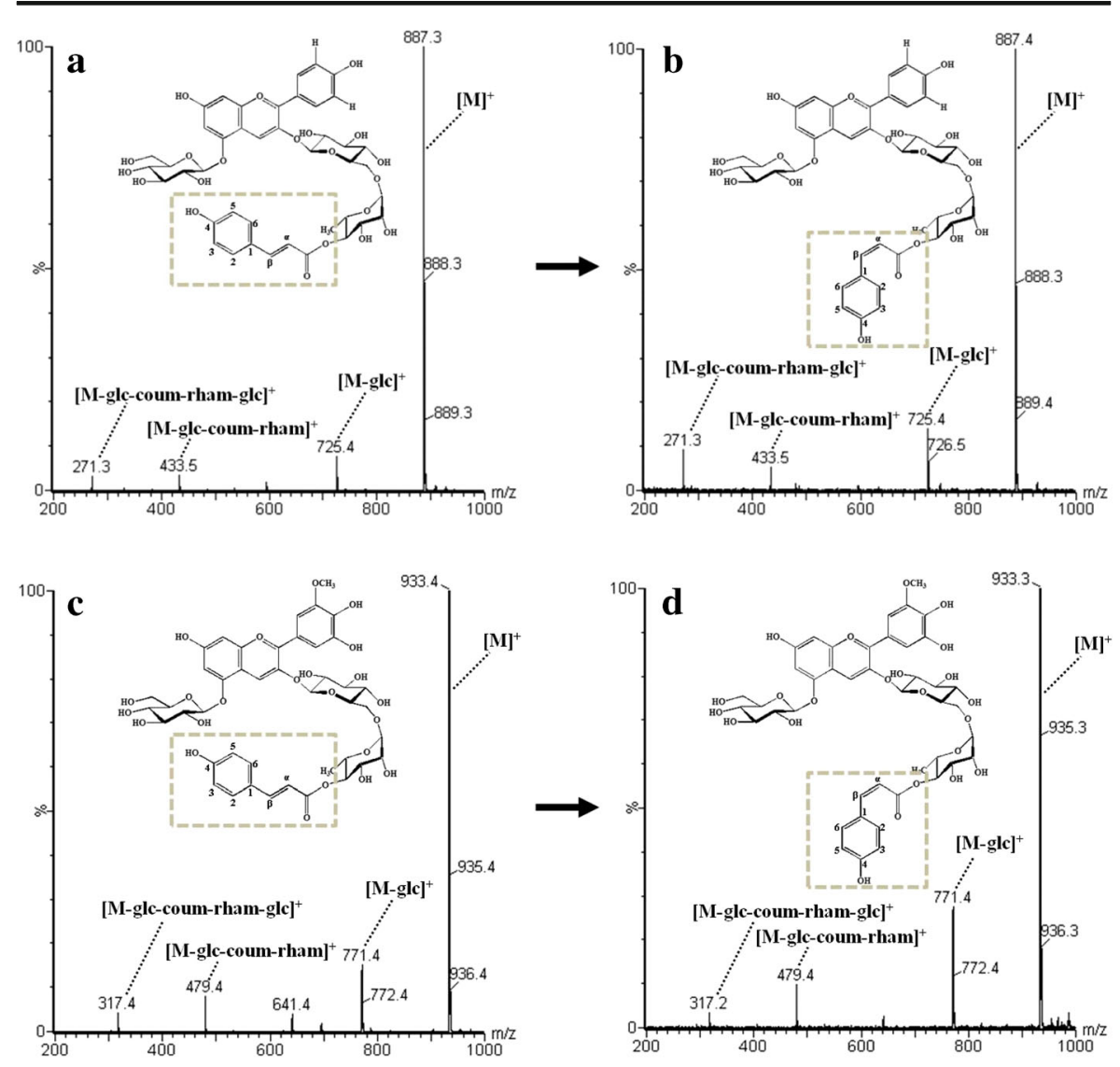

" glc, glucoside; rham, rhamnoside; coum, p-coumaroyl

Fig. 2 Structures and MS spectra showing the isomerization of major anthocyanin compounds isolated from extracts of Korean red- and purple-fleshed potatoes. a Pelargonidin 3-p-coumaroylrutinoside-5-glucoside. b Pelargonidin 3-cis-p-coumaroylrutinoside-5-glucoside. c Petunidin 3-p-coumaroylrutinoside-5-glucoside. d petunidin 3-cis-p-coumaroylrutinoside-5-glucoside

et al. 2008) (Fig. 2). Out of five cis isomers identified in the coloured potatoes, only a cis-pelanin (pelargonidin 3-cis-p-coumaroylrutinoside-5-glucoside) isomer has been reported in the literature (Mulinacci et al. 2008; Ieri et al. 2011). It was noted that the anthocyanins present in petunia petals and eggplants (Ichiyanagi et al. 2006; Slimestad et al. 1999) have similar structures to those in potatoes and they also contain cis isomers, especially in the natural, unprocessed state. Likewise, cis-pelanin and cispetanin isomers were identified as the principal anthocyanins in both red- and purplefleshed potatoes in their raw states. In addition, traces of acylated anthocyanins were detected, for which various kinds of cis isomers are expected to be found in colored potato cultivars by aglycone and acylation groups in the raw and/or processed forms.

Figure 3 shows representative HPLC chromatograms of individual anthocyanins in extracts from the tubers of colored potato cultivars (Hongyoung and Jayoung) and the breeding clone JJe08-11, in raw and baked forms. The number of individual anthocyanin species in these 5 potato samples was 8 in Hongyoung, 

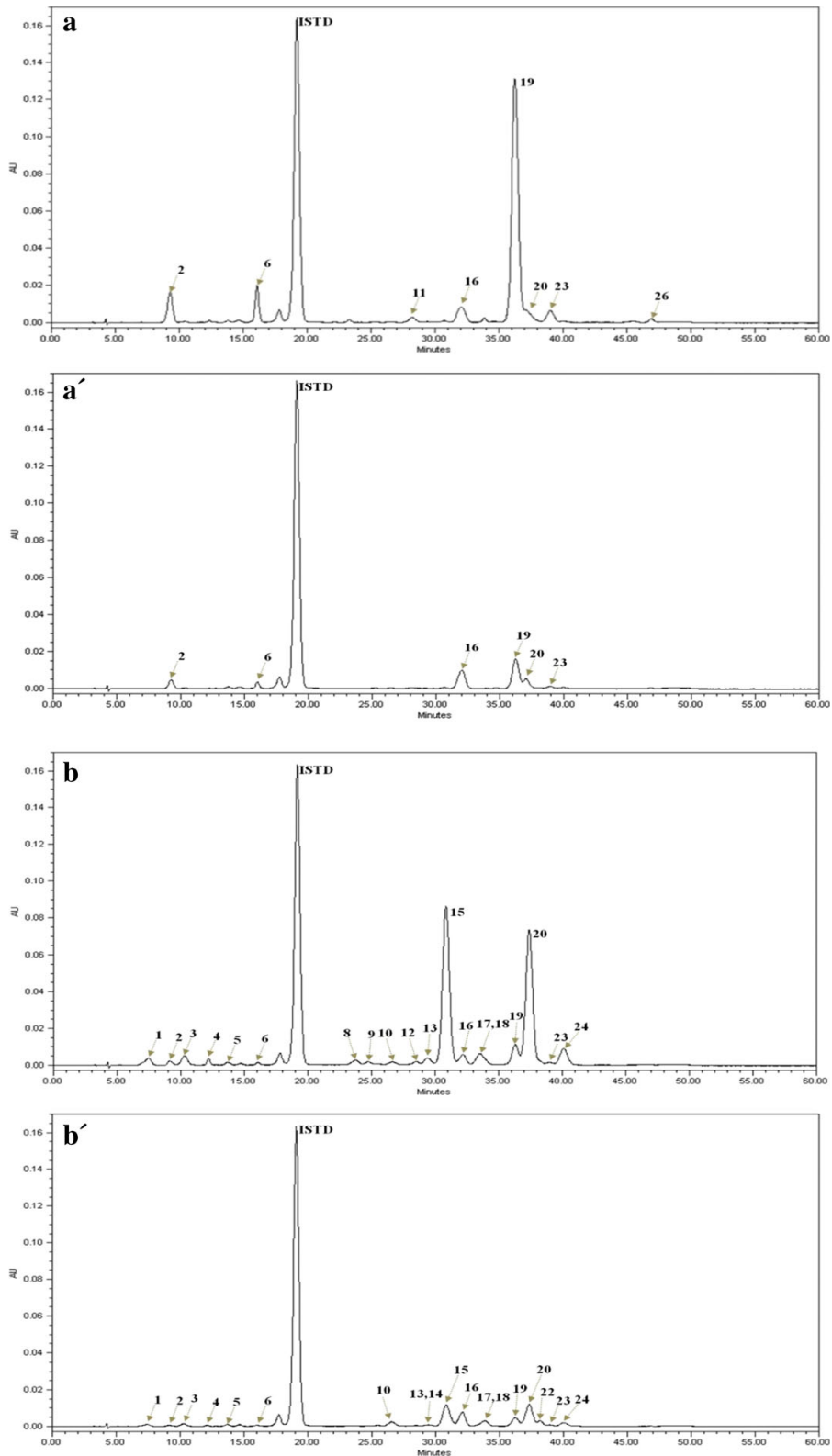

Fig. 3 HPLC-DAD chromatograms of individual anthocyanins extracted from the following Korean red- and purple-fleshed potatoes: Hongyoung (a: raw; a': baked), Jayoung (b: raw; b': baked), and JJe08-11 (c: raw; c': baked). ISTD (internal standard) $100 \mathrm{ppm}$ malvidin 3-glucoside (oenin). 1 pet-3-rut-5-glc, 2 pel-3-rut-5-glc, 3 peo-3-rut-5-glc, 4 cya-3-rut, 5 pet-3-rut, 6 pel-3-rut, 7 pet-3-cis-caf-rut-5-glc, 8 pet-3-caf-rut-5-glc, 9 del-3coum-rut-5-glc, 10 pet-3-cis-coum-rut-5-glc, 11 pel-3-caf-rut-5-glc, 12 isomer of pet-3-coum-rut-5-glc, 13 peo-3-caf-rut-5-glc, 14 pet-3-cis-fer-rut-5-glc, 15 pet-3-coum-rut-5-glc, 16 pel-3-cis-coum-rut-5-glc, 17 pet-3fer-rut-5-glc, 18 peo-3-cis-coum-rut-5-glc, 19 pel-3-coum-rut-5-glc, 20 peo-3-coum-rut-5-glc, 21 mal-3-coumrut-5-glc, 22 isomer of peo-3-coum-rut-5-glc, 23 pel-3-fer-rut-5-glc, 24 peo-3-fer-rut-5-glc, 25 mal-3-fer-rut-5glc, 26 pel-3-coum-rut, pel pelargonidin, cya cyanidin, peo peonidin, del delphinidin, pet petunidin, mal malvidin, coum p-coumaroyl, caf caffeoyl, fer feruloyl, rut rutinoside, glc glucoside 

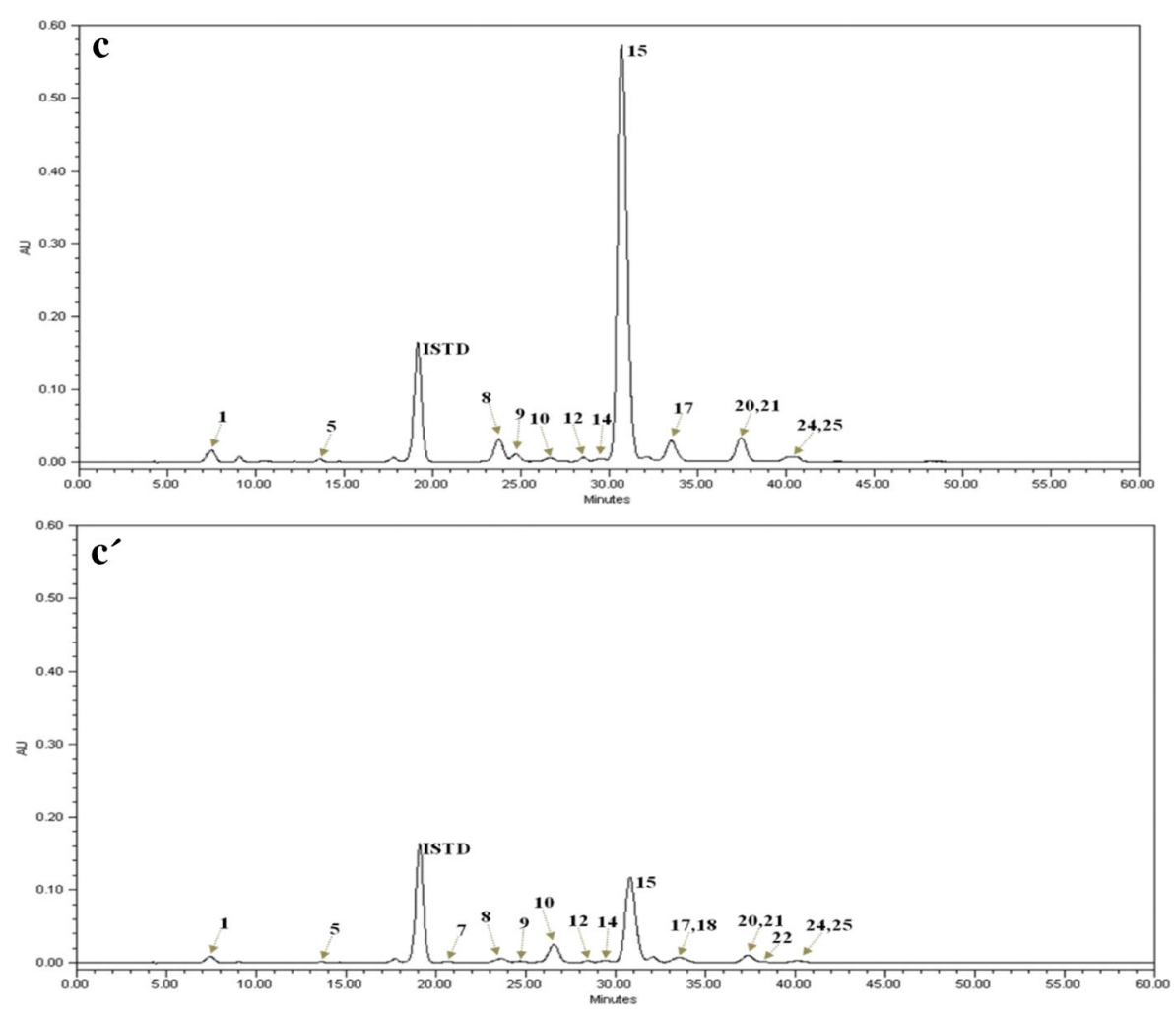

Fig. 3 (continued)

21 in Jayoung, and 16 each in JJe08-11, DJ12X-5, and JJe08-43. The largest number of potato anthocyanin species in the European purple-fleshed potato cultivar Shetland Black similar to Jayoung was 8-11 (Hillebrand et al. 2009; Ieri et al. 2011). Interestingly, in the present investigation, Jayoung exhibited various anthocyanins, including most compounds seen in other red- and purple-fleshed potato samples. Hence, it is expected to play an important role as a source of anthocyanins. The isolated individual anthocyanins were eluted in the following order like the patterns of previous results: delphinidin, cyanidin, petunidin, pelargonidin, peonidin, and malvidin for aglycones (Lachman et al. 2012) and caffeic acid, $p$-coumaric acid, and ferulic acid for acylation groups (Mulinacci et al. 2008; Ieri et al. 2011). The five cis isomers always appeared before their corresponding trans isomers in the pattern of trans anthocyanins (Downey and Rochfort, 2008). In particular, the cis-pelanin isomer has been reported in redfleshed potato cultivars such as 'Highland Burgundy Red' and 'Bamberger Hornchen' and is differentiated from trans-pelanin only by the elution order (Mulinacci et al. 2008; Ieri et al., 2011). Likewise cis-nasunin (delphinidin 3-cis$p$-coumaroylrutinoside-5-glucoside) has been reported to appear before transnasunin (delphinidin 3-trans-p-coumaroylrutinoside-5-glucoside) as major anthocyanins in eggplant (Ichiyanagi et al. 2006). However, another presumed isomer of petanin appeared before the cis isomer, and another presumed isomer of peonanin appeared after the trans isomer. 


\section{Anthocyanin Composition in Raw Korean Red- and Purple-Fleshed Potato Tubers}

Table 2 shows the anthocyanin concentration and composition of Korean red- and purple-fleshed potatoes according to their state (i.e., raw, baked, and steamed). These results were compared with the variations reported in 13 European colored potato varieties (Ieri et al. 2011) taking into account the dry-to-fresh-weight ratios (15.529.9\%). Red-fleshed Hongyoung potatoes showed an average anthocyanin concentration of $40.7 \mathrm{mg} / 100 \mathrm{~g}$ tuber dry weight (DW) in the raw state, of which pelargonidinand peonidin-based anthocyanins accounted for 97.5 and $2.5 \%$ respectively. The main anthocyanin was identified as pelanin, at $74.7 \%$ or $30.4 \mathrm{mg} / 100 \mathrm{-g}$ DW. Hongyoung exhibited an anthocyanin composition similar to the European red-fleshed potato cultivar Highland Burgundy Red, 33 red-fleshed potato cultivars (Solanum tuberosum and S. stenotomum), and 17 red-fleshed potato breeding clones (Mulinacci et al. 2008; Rodriguez-Saona et al. 1998; Brown et al. 2003).

The purple-fleshed cultivar Jayoung showed an average anthocyanin concentration of $50.8 \mathrm{mg} / 100$-g DW in the raw state, consisting of $0.8 \%$ cyanidin, $0.4 \%$ delphinidin, $8.3 \%$ pelargonidin, $43.9 \%$ peonidin, and $46.6 \%$ petunidin. The main anthocyanins were identified as peonanin $(34.1 \%, 17.3 \mathrm{mg} / 100 \mathrm{~g} \mathrm{DW})$ and petanin $(40.4 \%$, $20.5 \mathrm{mg} / 100 \mathrm{~g} \mathrm{DW})$. Jayoung exhibited a similar anthocyanin composition to the European purple-fleshed potato cultivar Shetland Black in its raw state (Hillebrand et al. 2009; Ieri et al. 2011).

Although JJe08-11, DJ12X-5, and JJe08-43 (purple-fleshed potato breeding clones) exhibited similar anthocyanin compositions, with average anthocyanin concentrations ranging between 21.9 and $163.4 \mathrm{mg} / 100 \mathrm{~g} \mathrm{DW}$ in the raw state, JJe08-11 had the highest anthocyanin concentration of the three. The proportions of the individual anthocyanins in these cultivars were $0.9-2.3 \%$ for delphinidin, $2.3-4.0 \%$ for malvidin, $1.8-3.8 \%$ for peonidin, and 91.1-93.2\% for petunidin. The main anthocyanin was identified as petanin (69.6-78.8\% of the total), ranging from 15.5 to $128.8 \mathrm{mg} / 100-\mathrm{g} \mathrm{DW}$ in the breeding clones. JJe08-11, DJ12X-5, and JJe08-43 (purple-fleshed) exhibited similar anthocyanin composition to the European purple-fleshed potato cultivars Salad Blue and Valfi, 28 purple-fleshed potato breeding clones, and 4 other purple-fleshed potato cultivars (Lachman et al. 2011; Jansen and Flamme 2006; Brown et al. 2003).

\section{Changes in Composition due to Processing}

Some earlier reports suggest that the total anthocyanin content of coloured potato cultivars and breeding clones decreases to 64.6-79.4\% when baked, 83.3-88.7\% when steamed, and 16-29\% when boiled or microwaved (Mulinacci et al. 2008; Nayak et al. 2011). In contrast, in other reports, some red- and purple-fleshed potato cultivars tended to have higher anthocyanin content when boiled, steamed, baked, or microwaved than when raw (Lachman et al. 2012). The susceptibility of potato cultivars to heat, light, and $\mathrm{pH}$ changes increased with an increase in the number of acyl groups present in their anthocyanins (Bakowska-Barczak, 2005). Moreover, the rate of decrease in anthocyanin content depends on the cultivar and processing conditions.

In line with the results above, individual and total anthocyanin concentrations decreased when potatoes were subjected to various processing conditions (Table 2). 


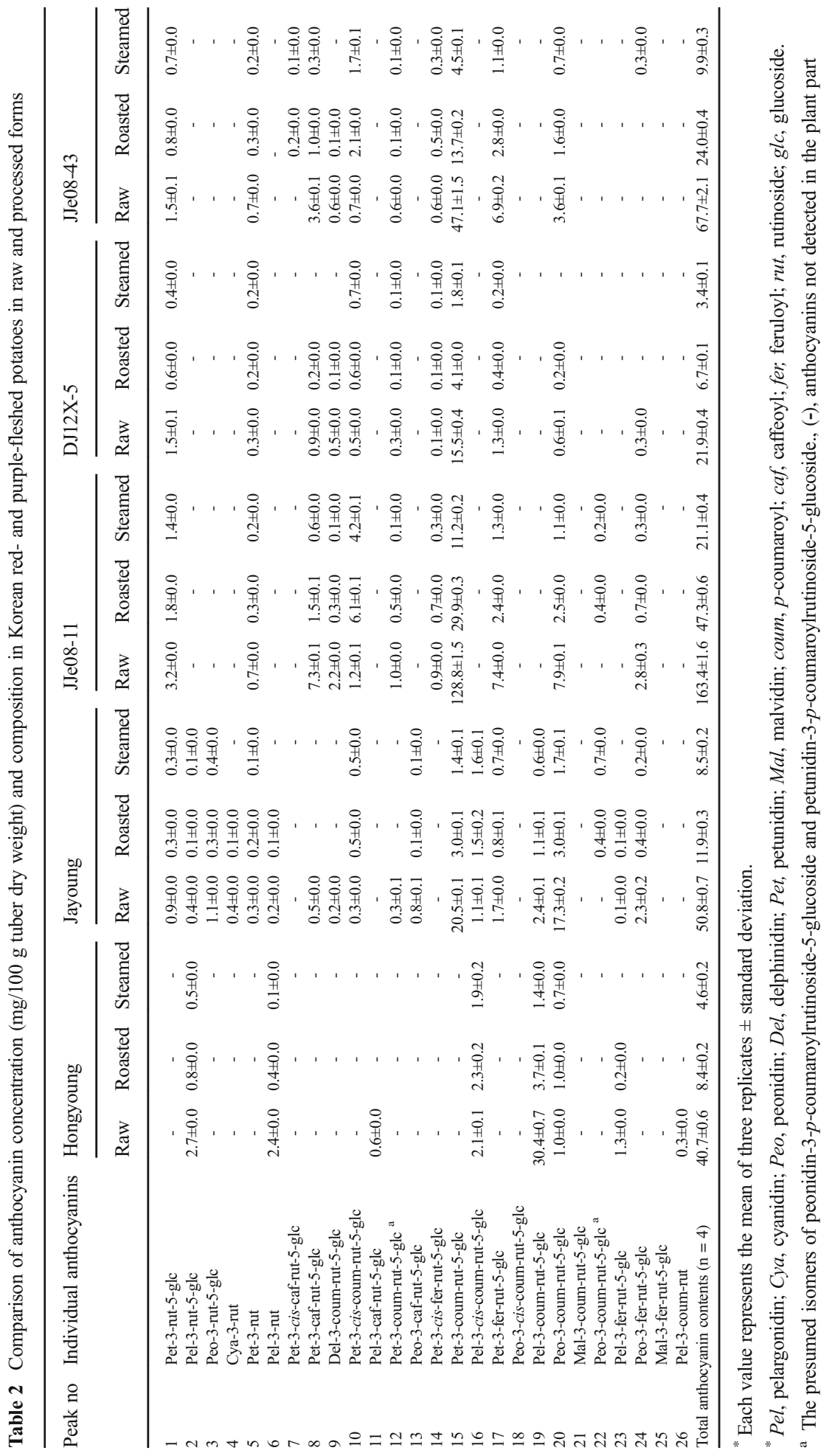


At the same time, the conversion of trans into cis occurred due to the structural change of acylated hydroxycinnamic acid (Fig. 2). Red- and purple-fleshed potatoes possessed large amounts of trans isomers in the raw state, in which pelanin and petanin were the principal anthocyanins, with their corresponding cis isomers increasing when baked or steamed (Table 2). In particular, the amount of cis-petanin in JJe08-11 increased over five times from 1.2 to $6.1 \mathrm{mg} / 100 \mathrm{~g}$ DW when baked (Table 2). Another presumed isomer (peak 12) of petanin existed in the raw state but slightly decreased when baked, while another presumed isomer (peak 22) of peonanin did not exist in the raw state but appeared when baked. In JJe08-11, DJ12X-5, and JJe08-43, petunidin 3-ciscaffeoylrutinoside-5-glucoside and cis-peonanin were not observed in the raw state because of small amounts of trans isomers, but they appeared when baked. In contrast, petunidin 3-cis-feruloylrutinoside-5-glucoside gradually decreased when baked or steamed. Unusually, in Jayoung, petunidin 3-cis-feruloylrutinoside-5-glucoside was produced in a mixed form when peonidin 3-caffeoylrutinoside-5-glucoside had almost disappeared.

\section{Partial Least Square Discriminant Analysis}

Recently, PLS-DA has been used to rapidly discriminate or detect differences in a wide variety of food, medicine, and agricultural products. PLS-DA is much better for distinguishing the characteristics of predefined groups (Perez-Enciso and Tenenhaus, 2003) than PCA, and its VIP (variable importance in the projection) is a variable that influences cluster formation. VIP is deemed significant when the value is greater than 1. Metabolomic data can be visualized by plotting principal component scores through alignment and standardization procedures. Figure 4 shows the overall patterns, variation, and cluster formation based on the anthocyanin profiles of colored potato samples under different types of processing in Table 2. When the correlations in anthocyanin composition and concentration between two tuber color groups were mapped by plotting PLS-DA scores, the red- and purple-fleshed potato groups formed different clusters, and the red-fleshed potato group in particular formed different clusters according to the type of processing (Fig. 4a). Principal component (PC) 1 and PC 2 accounted for $64 \%$ of the total variation as 52 and $12 \%$, respectively. The VIP value showed a high importance level (1 or higher) for P2 (pelargonidin 3-rutinoside-5glucoside, 1.27), P6 (pelargonidin 3-rutinoside, 1.22), P1 (petunidin 3-rutinoside-5glucoside, 1.21), P3 (peonidin 3-rutinoside-5-glucoside, 1.21), P16 (cis-pelanin, 1.15), P19 (pelanin, 1.12), P10 (cis-petanin, 1.11), and P15 (petanin, 1.06) (Fig. 4b). The colored potato anthocyanins were represented by pelargonidin derivatives for redfleshed potatoes and petunidin derivatives for purple-fleshed potatoes. Pelargonidinbased anthocyanins were more dominant than petunidin-based anthocyanins. Further, non-acylated anthocyanins, which constitute the majority of coloured potato anthocyanins, were highly involved in clustering, and cis and trans isomers of P19 and P15, the principal anthocyanins in both red- and purple-fleshed potatoes, were also involved in clustering (Fig. 4c). In particular, P16 and the cis isomer of P19 had an important impact on cluster formation. Although two clusters were formed for Jayoung and for JJe08-11, DJ12X-5, and JJe08-43 (Fig. 4c), Jayoung formed different clusters based on the types of processing. PC 1 and PC 2 accounted for $69 \%$ of the total variation, representing 43 and 26\%, respectively. As indicated in Table 2, JJe08-11, JJe08-43, and 
a

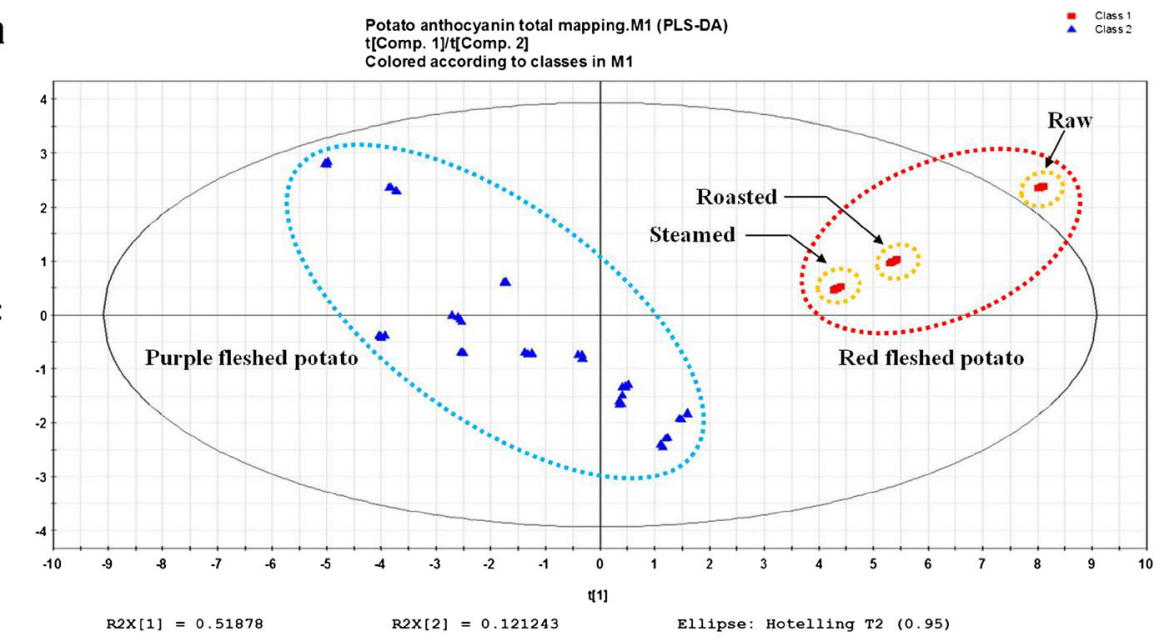

b

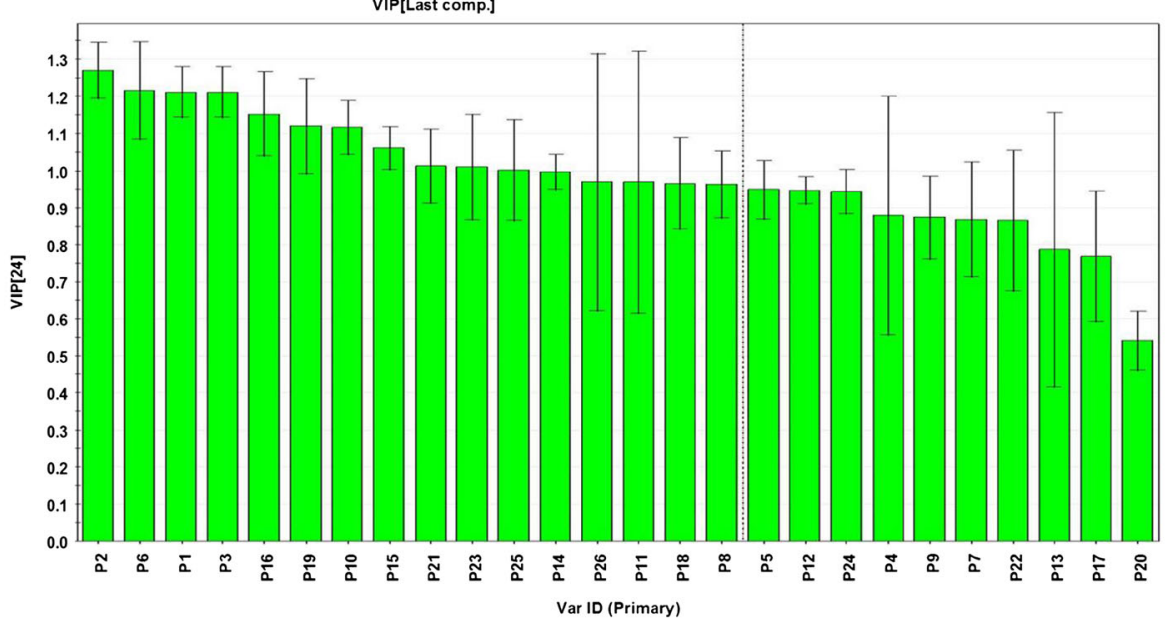

Fig. 4 Principal components 1 and 2 of the PLS-DA results obtained from anthocyanin profiling of Korean red- and purple-fleshed potato cultivars. a Total map on all samples (red box: red-fleshed potato; blue triangle: purple-fleshed potato). b Influence of 26 variables (potato anthocyanins: P1-P26) for the total map (VIP variable importance in the prediction). c Specific map on purple-fleshed potato (black box: Jayoung; red triangle: JJe08-11; purple star: JJe08-43; green diamond: DJ12X-5). d Influence of 24 variables (potato anthocyanins: P1-P10 and P12-P25) for specific map (VIP) (Color figure online)

DJ12X-5 exhibited the same anthocyanin composition; they appear in order from left to right. Potatoes in each sample appeared in order from raw to baked and then steamed. Likewise, Jayoung potatoes were shown in the order of raw, baked, and steamed from right to left. The lowest anthocyanin concentration is shown in the highest position in the individual sub-clusters. The VIP value was greater than 1 for P22 (another isomer of peonanin, 1.41), P10 (cis-petanin, 1.21), P14 (petunidin 3-cis-feruloylrutinoside-5glucoside, 1.20), P7 (petunidin 3-cis-caffeoylrutinoside-5-glucoside, 1.19), P9 (delphinidin 3-p-coumaroylrutinoside-5-glucoside, 1.12), P16 (cis-pelanin, 1.10), P20 (peonanin, 1.08), and P24 (peonidin 3-feruloylrutinoside-5-glucoside, 1.07) (Fig. 4d). 

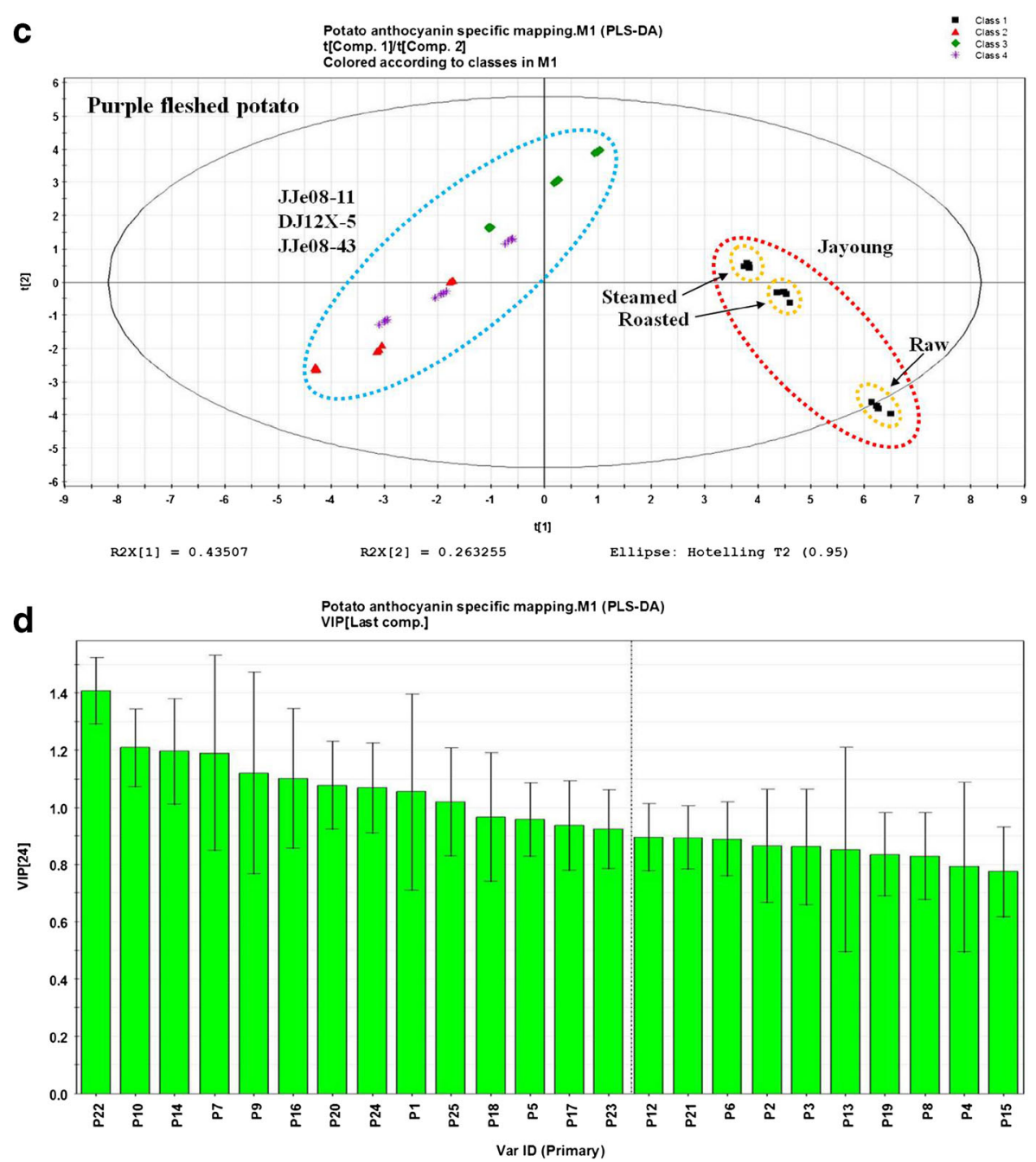

Fig. 4 (continued)

The results shown in Fig. 4c are interpreted in connection with the VIP, which benefitted interpretation of the findings provided in Table 2. They reflected the consistent but specific patterns of cis isomers in baked or steamed potatoes and indicated that most cis isomers were involved in clustering. P22, P10, and P14 were found to play important roles in clustering the processed forms of Jayoung. It is unusual that P14 was not found in Jayoung (Table 2) but appeared when baked or steamed and P20 and P24 clearly distinguished the two purple potato groups (Jayoung vs. JJe08-11, DJ12X-5, and JJe08-43), because none of those compounds occurred in combination with malvidin-based anthocyanins. These findings suggest that cis isomers were highly involved in clustering and played an important role as a biomarker under diverse potato processing conditions. The number of final clusters formed corresponds to the chromatograms of the three anthocyanin patterns presented in Fig. 3. 


\section{Conclusions}

Anthocyanin concentration, composition, and the effect of steaming and baking in tubers of Korean red- and purple-fleshed potato cultivars and breeding clones have been evaluated using LC-DAD-ESI-MS with malvidin 3-glucoside as an internal standard. A chemical library of 24 potato anthocyanins including MS fragment ion patterns was compiled from published data for efficient identification of anthocyanins. On the basis of this information, 26 anthocyanins with a basic structure of 3-rutinoside or 3-rutinoside-5-glucoside were separated, of which 24 were confirmed and 2 unknown isomers were presumed. Remarkably, five $c i s$ isomers were identified, of which four, viz., cis-petanin, cis-peonanin, petunidin 3-cis-caffeolylrutinoside-5-glucoside, and petunidin 3-cis-feruloylrutinoside-5-glucoside, are reported for the first time in Korean red- and purple-fleshed potatoes. In a PLS-DA model, all cis isomers were shown to play a role as biomarkers in clustering on the type of processing conditions. Pelargonidin 3-p-coumaroylrutinoside-5-glucoside (pelanin), peonidin 3-pcoumaroylrutinoside-5-glucoside (peonanin), and petunidin 3-p-coumaroylrutinoside5-glucoside (petanin) were identified as the major anthocyanins and were shown to play a role as biomarkers in a PLS-DA model based on the color of the tubers. The total anthocyanin concentration of the colored potatoes was decreased by steaming and baking processes compared with their concentration in the raw tubers.

We conducted a PLS-DA multivariate analysis of the patterns of principal anthocyanins and determined the VIP values. Korean colored potatoes formed specific clusters depending on their genotype and processed form. Anthocyanins possess important biological activity profiles, and we concluded that the present investigation contributes to evaluating the structural activity relationships (SAR) of individual anthocyanins, thus enhancing the value of colored potatoes in functional food or medicine.

Acknowledgements This study was conducted with research support as part of the Rural Development Administration's agenda project (Project No. PJ01178704). The authors would like to express deep appreciation for the support.

Open Access This article is distributed under the terms of the Creative Commons Attribution 4.0 International License (http://creativecommons.org/licenses/by/4.0/), which permits unrestricted use, distribution, and reproduction in any medium, provided you give appropriate credit to the original author(s) and the source, provide a link to the Creative Commons license, and indicate if changes were made.

\section{References}

Azuma K, Ohyama A, Ippoushi K, Ichiyanagi T, Takeuchi A, Saito T, Fukuoka H (2008) Structures and antioxidant activity of anthocyanins in many accessions of eggplant and its related species. J Agric Food Chem 56:10154-10159

Bakowska-Barczak A (2005) Acylated anthocyanins as stable, natural food colorants—a review. Pol J Food Nutr Sci 14:107-116

Brown CR, Wrolstad R, Durst R, Yang CP, Clevidence B (2003) Breeding studies in potatoes containing high concentration of anthocyanins. Am J Potato Res 80:241-250

Castaneda-Ovando A, Pacheco-Hernandez ML, Paez-Hernandez ME, Rodriguez JA, Galan-Vidal CA (2009) Chemical studies of anthocyanins: a review. Food Chem 113:859-871 
Cho K, Cho K-S, Sohn H-B, Ha IJ, Hong S-Y, Lee H, Kim Y-M, Nam MH (2016) Network analysis of the metabolome and transcriptome reveals novel regulation of potato pigmentation. J Exp Botany 67:15191533

Downey MO, Rochfort S (2008) Simultaneous separation by reversed-phase high-performance liquid chromatography and mass spectral identification of anthocyanins and flavonols in Shiraz grape skin. J Chromatogr A 1201:43-47

Ezekiel R, Singh N, Sharma S, Kaur A (2011) Beneficial phytochemicals in potato-a review. Food Res Int 50:487-496

Fossen T, Ovstedal DO, Slimestad R, Andersen OM (2003) Anthocyanins from a Norwegian potato cultivar. Food Chem 81:433-437

Giusti MM, Rodriguez-Saona LE, Griffin D, Wrolstad RE (1999) Electrospray and tandem mass spectroscopy as tools for anthocyanin characterization. J Agric Food Chem 47:4657-4664

Hillebrand S, Naumann H, Kitzinski N, Kohler N, Winterhalter P (2009) Isolation and characterization of anthocyanins from blue-fleshed potatoes (Solanum tuberosum L.) Food Rev Global Sci Books 3:96-101

Ieri F, Innocenti M, Andrenelli L, Vecchio V, Mulinacci N (2011) Rapid HPLC/DAD/MS method to determine phenolic acids, glycoalkaloids and anthocyanins in pigmented potatoes (Solanum tuberosum L.) and correlations with variety and geographical origin. Food Chem 125:750-759

Ichiyanagi T, Terahara N, Rahman MM, Konishi T (2006) Gastrointestinal uptake of nasunin, acylated anthocyanin in eggplant. J Agric Food Chem 54:5306-5312

Jansen G, Flamme W (2006) Coloured potatoes (Solanum Tuberosum L.) - anthocyanin content and tuber quality. Genet Resour Crop Ev 53:1321-1331

Kong JM, Chia LS, Goh NK, Chia TF, Brouillard R (2003) Analysis and biological activities of anthocyanins. Phytochemistry 64:923-933

Kalt W, Blumberg JB, Mcdonald JE, Vinqvist-Tymchuk MR, Fillmore SAE, Graf BA, O’Leary JM, Milbury PE (2008) Identification of anthocyanins in the liver, eye, and brain of blueberry-fed pigs. J Agric Food Chem 56:705-712

Kang SC, Choung MG (2008) Comparative study on biological activities of colored potatoes, Hongyoung and Jayoung cultivar. Kor J Crop Sci 53:233-238

Kim HW, Kim JB, Cho SM, Chung MN, Lee YM, Chu SM, Che JH, Kim SN, Kim SY, Cho YS, Kim JH, Park HJ, Lee DJ (2012) Anthocyanin changes in the Korean purple-fleshed sweet potato, Shinzami, as affected by steaming and baking. Food Chem 130:966-972

Lachman J, Hamouz K, Orsak M, Pivec V, Hejtmankova K, Pazderu K, Dvorak P, Cepl J (2012) Impact of selected factors - cultivar, storage, cooking and baking on the content of anthocyanins in coloured-flesh potatoes. Food Chem 133:1107-1116

Lachman J, Hamouz K, Sulc M, Orsak M, Pivec V, Hejtmankova A, Dvorak P, Cepl J (2009) Cultivar differences of total anthocyanins and anthocyanidins in red and purple-fleshed potatoes and their relation to antioxidant activity. Food Chem 114:836-843

Mulinacci N, Ieri F, Giaccherini C, Innocenti M, Andrenelli L, Canova G, Saracchi M, Casiraghi MC (2008) Effect of cooking on the anthocyanins, phenolic acids, glycoalkaloids, and resistant starch content in two pigmented cultivars of Solanum tuberosum L. J Agric Food Chem 56:11830-11837

Nayak B, Berrios JJ, Powers JR, Tang J (2011) Thermal degradation of anthocyanins from purple potato (cv. Purple Majesty) and impact on antioxidant capacity. J Agric Food Chem 59:11040-11049

Park HJ, Jeon TW, Lee SH, Cho YS, Cho SM, Chang KS (2004) Studies on characteristics and stability of anthocyanin pigment extracted from Korean purple-fleshed potatoes. J Kor Soc Food Sci Nutr 33:1544 1551

Park YE, Cho HM, Lee HJ, Hwang YS, Choi SN, Lee SJ, Park ES, Lim JD, Choung MG (2007) Antioxidant and inhibition on angiotensin converting enzyme activity of colored potato extracts. Kor J Crop Sci 52: $447-452$

Park YE, Jeong JC, Cho HM, Hwang YS, Lee HJ, Choi SN, Lee SJ, Park ES, Ko EA, Kim NS, Lim JD, Choung MG (2008) Antimutagenic effect and cytotoxcity to human cancer cell of colored potato extracts. Kor J Crop Sci 53:75-84

Perez-Enciso M, Tenenhaus M (2003) Prediction of clinical outcome with microarray data: a partial least squares discriminant analysis (PLS-DA) approach. Hum Genet 112:581-592

Rodriguez-Saona LE, Giusti MM, Wrolstad RE (1998) Anthocyanin pigment composition of red-fleshed potatoes. J Food Sci 63:458-465

Rodriguez-Saona LE, Giusti MM, Wrolstad RE (1999) Color and pigment stability of red radish and redfleshed potato anthocyanins in juice model systems. J Food Sci 64:451-456

Slimestad R, Aaberg A, Andersen OM (1999) Acylated anthocyanins from petunia flowers. Phytochemistry 50:1081-1086 
Wu X, Beecher GR, Holden JM, Haytowitz DB, Gebhardt SE, Prior RL (2006) Concentration of anthocyanins in common foods in the United States and estimation of normal consumption. J Agric Food Chem 54: 4069-4075

Wang LS, Stoner GD (2008) Anthocyanins and their role in cancer prevention. Cancer Lett 269:281-290

Xu X, Li W, Lu Z, Beta T, Hydamaka AW (2009) Phenolic content, composition, antioxidant activity, and their changes during domestic cooking of potatoes. J Agric Food Chem 57:10231-10238

Zhao CL, Guo HC, Dong ZY, Zhao Q (2009) Pharmacological and nutritional activities of potato anthocyanins. Afr J Pharm Pharmacol 3:463-468 